\title{
Pathology and Pathogenesis of Progressive Multiple Sclerosis: Concepts and Controversies
}

Authors

Adrian-Minh Schumacher ${ }^{*}$, Christoph Mahler ${ }^{*}$, Martin Kerschensteiner

\author{
Affiliation \\ Institute of Clinical Neuroimmunology, Biomedical Center \\ and University Hospital, Ludwig-Maximilians Universität \\ München, Munich, Germany \\ Key words \\ multiple sclerosis, white matter pathology, grey matter \\ pathology, chronic inflammation, neurodegeneration \\ Bibliography \\ DOI https://doi.org/10.1055/s-0043-106704 \\ Published online: 2017 \\ Neurology International Open 2017; 1: E171-E181 \\ (c) Georg Thieme Verlag KG Stuttgart · New York \\ ISSN 2511-1795 \\ Correspondence \\ Prof. Dr. med. Martin Kerschensteiner \\ Institut für Klinische Neuroimmunologie \\ Biomedizinisches Zentrum und Klinikum der Universität \\ München \\ Ludwig-Maximilians Universität München \\ Grosshaderner Str. 9 \\ 82152 Martinsried \\ Germany \\ Martin.Kerschensteiner@med.uni-muenchen.de
}

\section{Introduction}

Multiple sclerosis (MS) is a common inflammatory disease of the nervous system that generally starts in young adulthood and over time frequently leads to progressive functional deficits. A characteristic of this still puzzling disease is that its clinical manifestation changes as it progresses. Although during the early phases of the disorder, most patients mainly experience relapses, on average after 10 to 15 years, this episodic relapsing-remitting phase develops into a progressive phase characterized by steadily increasing disability [1]. Why this transition happens and why approx. $15 \%$ of all patients enter this progressive phase without a preceding relapsing-remitting phase are questions which have yet to be answered completely. The importance of finding answers to these questions is pinpointed by the fact that although numerous medications are effective in treating relapsing-remitting MS, they re-

* The authors contributed in equally to this article.

\begin{abstract}
Multiple sclerosis (MS) is an inflammatory disease of the central nervous system that initially is often dominated by relapsingremitting neurological symptoms. With increasing disease duration these relapses are more and more superimposed by a progressive disease process that leads to an irreversible accumulation of motor, sensory and cognitive deficits. This progressive phase of MS is still only incompletely understood and by and large refractory to therapy. Here we aim to use recent pathological and pathomechanistic insights to outline a unifying concept of progressive MS. Based on this view of the disease we examine current controversies surrounding progressive MS. We discuss whether neurodegenerative or inflammatory processes drive progression, question whether the classification of primary and secondary progressive MS is all that useful and deliberate, which therapeutic strategies are best suited to limit the insidious neurological decline of progressive MS patients.
\end{abstract}

main partially or completely ineffective in the progressive stage of the disease. We will approach these questions by summarizing the major advances in the study of the pathology and pathogenesis of progressive MS, and then discuss which concepts and conclusions emerge from these findings.

\section{Pathology of Progressive Multiple Sclerosis}

In his 1868 lecture on the "Histologie De La Sclérose En Plaques" at the Hôpital de la Salpêtrière, J.M. Charcot described frequently-found periventricular demyelinating lesions as a histological hallmark of multiple sclerosis and a correlate of clinical relapses [2]. Histopathologically, the plaques usually show marked myelin loss, signs of acute axonal damage and reactive astrogliosis [3]. However, this pathological manifestation of multiple sclerosis changes as the disease progresses. The following sections will describe the focal and diffuse changes in the white and gray matter that char- 
acterize the pathological pattern of progressive MS (• Fig. 1). In particular, we will examine the extent to which focal and diffuse pathology are fundamentally different and how they can causally influence one another.

\section{Pathological Changes in the White Matter}

Traditionally, multiple sclerosis has been considered a disease of the white matter. Although focal demyelinating lesions have been known since Charcot's time and have long been the focus of MS research, recent histopathological and imaging studies also point to substantial lesion-independent changes in the otherwise macroscopically normal-appearing white matter (NAWM). The following will provide an overview of the essential focal and diffuse changes of white matter in the progressive stage of the disease.

\section{Focal pathology - chronic active and inactive lesions}

Active lesions mainly occur in the early relapse-remission stage and exhibit dense infiltration by macrophages, while progressive MS is characterized by chronic active and inactive lesions ( $>$ Fig. 2 ). Inactive lesions lack the signs of an acute inflammatory reaction and, with their hypocellular gliotic appearance, represent the "burned

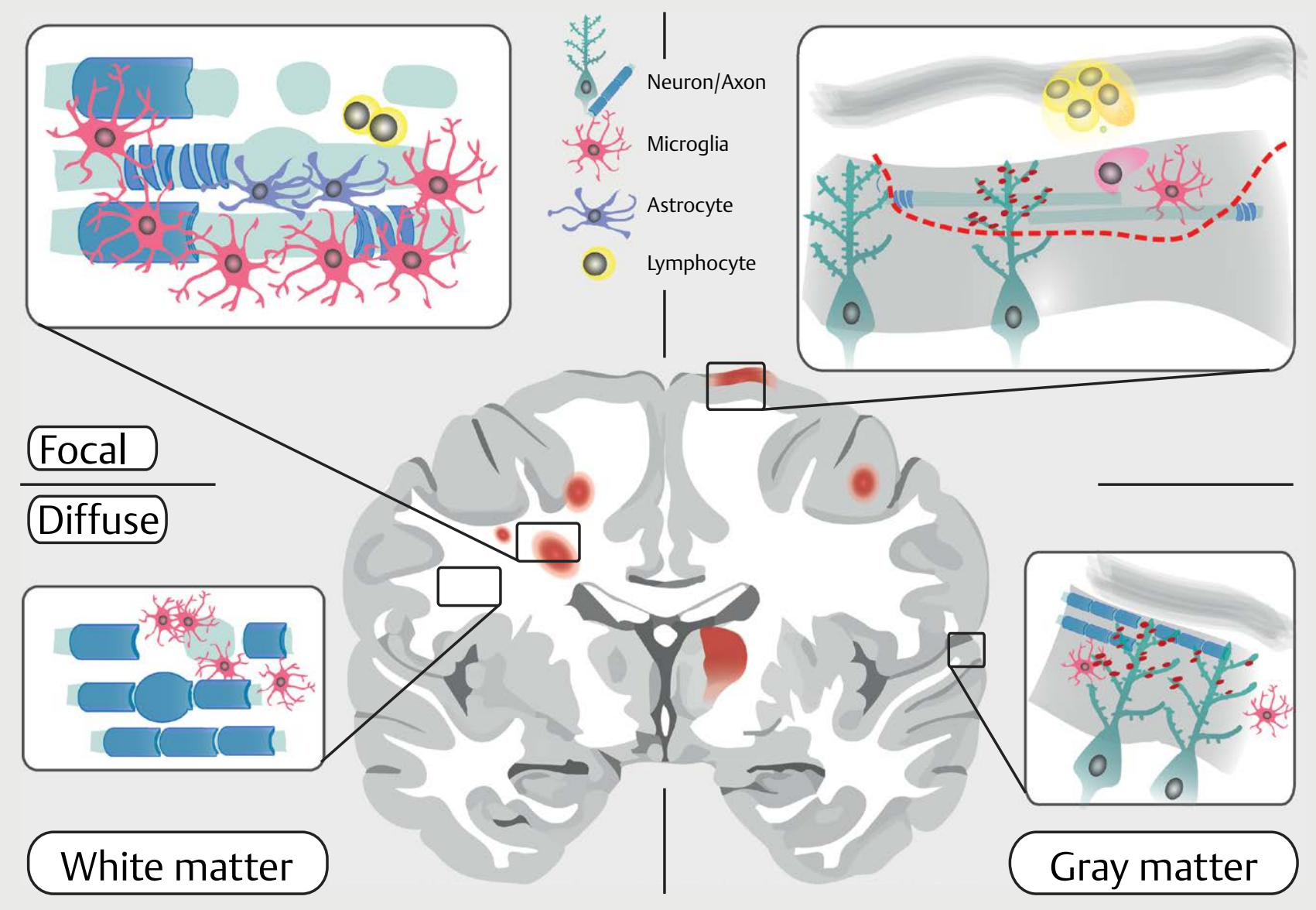

- Fig. 1 Illustration of characteristic focal and diffuse changes in white and gray matter in progressive multiple sclerosis.

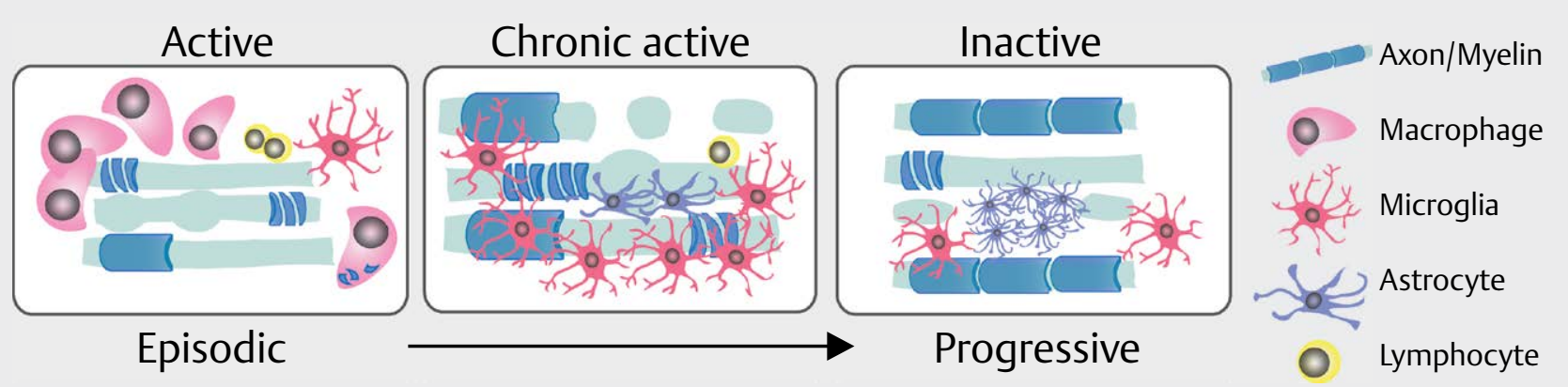

Fig. 2 Transformation of typical lesion patterns in the course of multiple sclerosis. 
out" end stage of an inflammatory lesion. On the other hand, chronic active lesions, which are found almost exclusively in the primary and secondary progressive types [4], show an active margin of microglia and macrophages. It is presumed that these "smoldering lesions" spread by means of a chronically lingering activation of microglia. Among other things, this idea is supported by a recent study, in which such chronically active lesions were tracked over time using 7-tesla MRI [5]. Paramagnetic "phase rings" were found in the marginal area of these lesions, presumably corresponding to the inflammatory microglial wall of chronically active lesions. In fact, post-mortem brain sections of lesions with such a phase ring histologically demonstrated a clear infiltration of activated and iron-laden macrophages as well as microglia in the margin of the lesion. Interestingly, if the paramagnetic phase rings persist in the peripheral region of the lesion, there is an increased hypointensity in the $\mathrm{T} 1$-weighted images, which is interpreted as progressive tissue damage.

While some of the chronic lesions of the white matter contribute to a slow progressive inflammatory damage of the CNS, this does not necessarily have to happen to all inflammatory MS lesions as reparative processes such as remyelination can be activated. Remyelinated lesions appear histopathologically as a "shadow plaque" due to the newly formed myelin, but the capacity to restore the myelin layer greatly varies among CNS regions and individual patients. Furthermore, although remyelinating foci can be found in all stages of the disease, they are encountered more frequently in the relapsing-remitting phase of MS. The reasons for this decline of endogenous repair capacity in the course of the disease are still incompletely understood. One possible explanation for these differences might be the loss of oligodendrocyte precursor cells in the course of multiple demyelinating-remyelinating episodes, thus exhausting this cellular reserve. On the other hand, a comparatively high density of such precursor cells is still found in many demyelinating lesions. So it appears more likely that the failure of remyelination is related to disturbed maturation of these cells possibly as a result of the chronic inflammatory reaction and astrocytic gliosis, or that the axons damaged by inflammation are themselves responsible for preventing successful remyelination by expressing inhibitory molecules [6]. Both the age of the lesion as well as the age of the patient play a role in this. Studies using experimental models show that the capacity for remyelination decreases with age. In these models, remyelination capacity can also be restored in old mice by transferring "young" inflammatory cells; therefore age-dependent changes in the immune system must play an important causative role [7]. In line with this finding, the chronically destructive lesions with a paramagnetic phase ring mentioned above are almost exclusively found in patients in advanced age [5].

\section{Diffuse pathology - microglia activation and axon loss}

In addition to the occurrence of focal white matter lesions, patients with progressive MS also exhibit evidence of diffuse inflammatory activity ( $\triangleright$ Fig. 1). On the one hand, this is manifested by a lowgrade but wide-spread accumulation of T-lymphocytes in the CNS; on the other hand, perivascular infiltrates made up primarily of mononuclear cells form around small veins [8]. In addition to this infiltration of inflammatory cells into the CNS, there is also diffuse microglia activation, as evidenced by the widespread formation of nests of activated microglia cells [9]. Recent PET studies, in which activated microglia and macrophages in patients could be demonstrated in vivo employing radioactive markers support these observations. In these studies, MS patients exhibit raised inflammatory activity even in normal-appearing white and gray matter (NAWM and NAGM, respectively) compared to controls. If one compares the different forms of MS regarding the extent of diffuse microglia activation, the PET studies concur with the histological findings of particularly pronounced microglia activation in progressive MS [10]. In normal-appearing white matter, there is not just a migration of inflammatory cells and activation of microglia, but also neuronal damage. First and foremost, axons are affected which exhibit increased focal swellings or are already fragmented. These swollen and fragmented axons are found throughout the normal-appearing white matter, with only a slight accumulation around demyelinating lesions [9]. Thus suggesting that diffuse axon damage is not entirely due to propagated degeneration out of focal white matter lesions. This is also confirmed by the fact that the quantity of focal white matter lesions does not show a clear correlation to the extent of diffuse axonal damage in normal-appearing white matter [9]

\section{Pathological Changes in the Gray Matter}

Diffuse and focal alterations of gray matter contribute not only to the pathological, but also to the clinical manifestation of multiple sclerosis. In fact, it is precisely the pathological changes of gray matter, including its atrophy, which at this stage determine the prognosis of the patient and, in particular, the appearance of cognitive symptoms [11].

\section{Focal pathology - demyelination and meningeal infiltration}

Already during the relapsing-remitting phase and increasingly with the onset of the progressive phase of the disease, demyelinated lesions occur not only in the white but also in the gray matter ( Fig. 1). They affect among others the cortex, where they occur more frequently in the sulci and in particular in the deeply furrowed or invaginated brain regions, such as the cingulate cortex and insular cortex [12]. Demyelinating gray matter lesions are found not only in the cortex, but also in the deeper brain regions such as the thalamus, the basal ganglia, the hippocampus as well as in the spinal cord $[13,14]$. These gray matter lesions contain only few infiltrating macrophages and T-cells which suggests that other factors drive the damage process [15]. Meningeal inflammation and in particular the formation of meningeal follicles play an important role in this [16]. These follicles predominantly consist of perivascular B-cells, T-cells, macrophages and plasma cells, and in their location, as described for subpial lesions, show a preference for the deep gyri and sulci possibly due to the lower cerebrospinal fluid flow in these regions [12]. Indeed, a subpial lesion is frequently found in the cortex near a B-cell follicle [17]. The pathological and clinical significance of meningeal follicles has been confirmed by a series of studies demonstrating that patients with meningeal B-cell follicles exhibit overall greater local inflammation and tissue damage [16]. The release of inflammation-promoting factors from B-cell follicles 

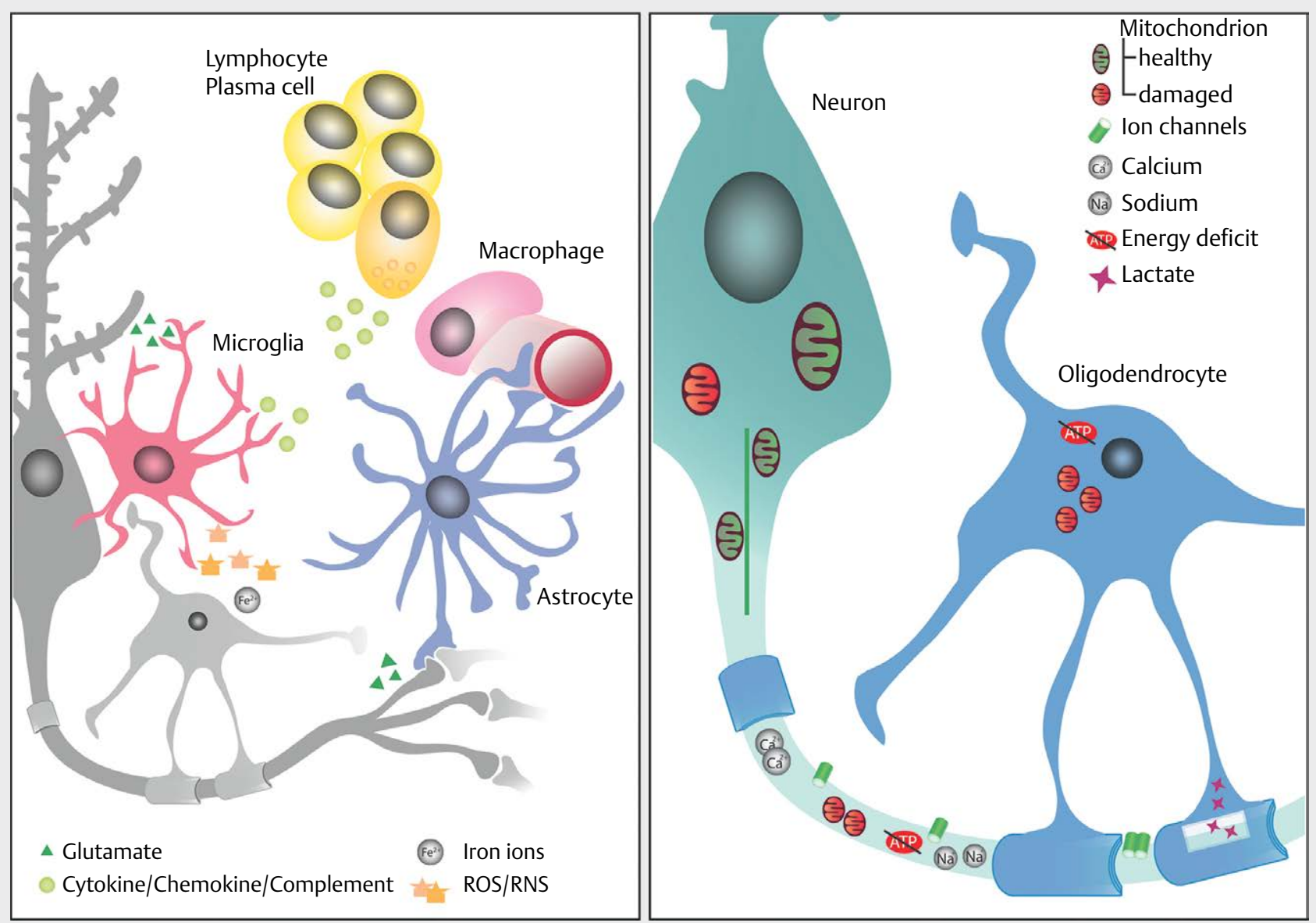

- Fig. 3 Illustration of the most important pathomechanical changes during progressive multiple sclerosis with respect to chronic inflammatory reaction (left) and the structurally and functionally destabilized nervous system (right).

can stimulate parenchymal inflammatory cells, in particular macrophages and microglia cells which, in turn, can promote local demyelination and neuronal damage via e. g. oxidative damage mechanisms $[12,18]$. Last but not least, meningeal inflammation also affects the clinical course of MS patients, as patients with histopathologically detected B-cell follicles are younger on average at the onset of disease, and the progression of disability and death occur earlier [19].

\section{Diffuse pathology - neurodegeneration and synapse loss}

Magnetic resonance imaging (MRI) examinations demonstrate that disease progression in MS results in accelerated atrophy of gray matter, which is clinically associated with the patient's increased cognitive decline [20]. Likewise in the spinal cord, the atrophy of the gray matter correlates with the extent of motor deficits, especially in the lower extremities. Atrophy of the gray matter of the spinal cord is an independent predictor of disease progression and is unrelated to white matter atrophy [21]. A comparative histopathological and MRI examination of brain slices shows that the main pathological basis of this atrophy are neurodegenerative processes, mainly the loss or decrease in the size of the cortical nerve cells and the loss of axonal connections [22]. Interestingly, cortical myelin density shows no correlation to local atrophy, which suggests that a significant component of these neurodegenerative processes is independent of the formation of focal demyelinating lesions in the cortex. Recent studies of synaptic pathology provide further evidence for the presence of such diffuse neurodegenerative processes in the gray matter of MS patients. Thus, a high-resolution reconstruction of individual cortical projection neurons revealed a pronounced synaptic loss, encompassing almost half of the dendritic synapses in both demyelinated and normal-appearing areas of the cortex [23]. Similar primarily lesion independent synaptic changes were also observed in the cerebellar dentate nucleus [24].

To date this synapse loss has not been sufficiently explained. On the one hand, there is evidence of direct inflammation-mediated synaptic damage, which is triggered, for example, by activated microglia cells or diffuse infiltrating T-cells and macrophages [25]. Indeed in the hippocampus and cerebellum of MS patients, synapses are found which are enclosed by microglia and astrocyte processes, and are thus detached from their post-synaptic neuron [24]. The retrograde degeneration of neurons is a second mechanism of diffuse neuronal damage in the gray matter. This type of anterograde or retrograde neurodegeneration, after transection of the associated axon in the white matter, can be traced using cytoplas- 
mically enriched, phosphorylated neurofilaments in neurons. These phosphorylated neurofilaments have been shown to occur more frequently in the gray matter in MS patients in close proximity to white matter lesions. On the other hand, there are few hints for retrograde neurodegeneration in subpial lesions which are more remote from the white matter [12]. The causes of cortical neurodegeneration are therefore likely due to a variety of mechanisms, which have a varying influence, depending on the location and type of lesion. If in addition to the cortex, entire hemispheres are examined for regions with pronounced retrograde neurodegeneration, the deep cerebral nuclei, especially the globus pallidus and the thalamus, appear particularly prominent. Within these brain nuclei, mainly neurons topographically related to lesions in the white matter are affected. This topographical association of neuronal damage in brain nuclei and lesions of the white matter further supports the concept of propagated neurodegeneration [12].

In summary, both pathological and imaging findings emphasize the significance of diffuse neurodegenerative processes for progressive MS. However, the mechanisms driving this degeneration of the gray matter are difficult to delineate solely based on clinical findings. Consequently, we would like to include experimental investigations into our considerations and thereby try to understand the pathomechanisms underlying progressive MS.

\section{Concepts of Pathogenesis}

We will focus on the following 2 aspects in our consideration of the mechanisms that promote progressive tissue damage in multiple sclerosis. On the one hand, we will discuss the chronic inflammatory reaction, which supports the formation of a toxic milieu in the central nervous system behind a largely closed blood-brain barrier. On the other hand, we will consider the nervous system itself, which has lost its homeostatic equilibrium due to prior damage, and is now all the more sensitive to inflammatory insults.

\section{Chronic Inflammation and Toxic}

\section{Environment}

In the above-described chronic lesions, despite moderate inflammatory activity, there are clear indications of a milieu, which promotes progressive degeneration of neurons, axons and oligodendrocytes and thus clinical progression of the disease. In addition to classical mediators of the immune system, this environment also contains increased concentrations of oxidants and iron ions as well as the excitatory neurotransmitter glutamate (see - Fig. 3). In the following sections, we will discuss how these alone or in combination can drive the decline of nerve and glial cells.

\section{Inflammatory mediators: cytokines, chemokines and complement factors}

As shown above, in the course of multiple sclerosis diffuse neuronal damage as well as extensive subpial demyelination of the cortex occur without pronounced local infiltration of immune cells. A possible cause for this diffuse damage are soluble factors secreted by immune cells with the potential to damage nerve cells and myelin even across longer distances. These include immune mediators, such as antibodies, cytokines and chemokines as well as other cytotoxic factors (e.g., matrix metalloproteinases, granzymes, and perforin). Producers of such soluble toxic factors can be meningeal inflammatory infiltrates including B-cell follicles, which have immediate access to the CSF. Indeed increased concentrations of inflammatory mediators such as tumor necrosis factor (TNF) and interferon-y have been detected in the CSF of patients with progressive MS [26, 27]. Interestingly, local application of these mediators into the cortex or the subarachnoidal space in an animal model of multiple sclerosis has led to a similarly extensive subpial demyelination as found in human cortical lesions [28]. However, extensive demyelination, encompassing both the ipsilateral and contralateral hemisphere, appears only in those animals in which an autoreactive immune response was triggered by previous immunization with myelin antigens. This indicates that cytokines can have their full pathological effect only in conjunction with components of the adaptive immune response, such as myelin-reactive antibodies.

In addition to meningeal infiltrates, however, resident cells of the brain parenchyma can also contribute decisively to a chronic inflammatory reaction. Microglia cells, which secrete cytokines and chemokines during chronic activation, sustain the inflammatory reaction. In addition, astrocytes are gaining more and more attention. As regulators of brain metabolism, neuronal transmission and the blood-brain barrier, these cells play a central role for CNS homeostasis. In response to damage to the nervous system, these cells become "reactive" and can assume different phenotypes [29]. While after a stroke, for example, reactive astrocytes acquire a neuroprotective phenotype, the same cells assume a neurotoxic phenotype under the influence of inflammatory mediators secreted by chronically activated microglia cells and can then directly trigger damage to neurons and oligodendrocytes [30]. However, astrocytes are not only possible agents in tissue damage, but also play an important role in immune regulation. This role also seems to be decisively determined by their phenotype. On the one hand, astrocytes can produce lactosylceramides and thus support their pro-inflammatory (neurotoxic) mode, which promotes the further recruitment of peripheral phagocytes via cytokines [31]. On the other hand, they can also be important producers of interferon- $\beta$, which exerts its anti-inflammatory effects on microglia and macrophages. In addition to immune regulation, activated astrocytes are also directly involved in the function of the nervous system as shown in experimental models. Through the release of TNF- $\alpha$ they modify the signals of excitatory synapses in the hippocampus [32]. Taken as a whole, these findings suggest that astrocytes are able to sustain a chronic tissue-damaging inflammatory response. Histopathologically, there are also indications that astrocytes express markers of a pro-inflammatory and neurotoxic phenotype in MS lesions [30,31]. However, it must be kept in mind that cell phenotypes can significantly differ between humans and mice, and thus the expression of individual characteristic marker proteins does not automatically allow the transfer of all mechanistic assessments from animal models to humans.

In the inflamed nervous system, the function and structure of synapses is impaired not only by pro-inflammatory cytokines such as TNF- $\alpha$, but also by components of the complement system. These complement proteins play a significant role as mediators of synapse removal in the development of the nervous system, in the 
aging brain and in neurodegenerative disease. Complement-tagged synapses are detected by microglia cells and then removed [33]. Recent studies have shown that the gray matter of progressive MS patients also exhibits increased activation of the complement system in a spatial relationship with microglia cells and synaptic pathology [34], which might indicate that in the inflamed brain mechanisms of synaptic degradation known from development are being reactivated.

\section{Oxidative stress and fenton reaction}

In addition to the release of cytokines, chemokines and complement factors, the accumulation of reactive oxygen and nitrogen species (ROS/RNS) occurs in acute or chronically inflamed tissue. These free radicals have high oxidative potential and in experimental models of MS cause damage to nerve and glial cells [35]. In multiple sclerosis lesions, oxidized DNA and phospholipids are found in both highly active and chronically active lesions, indicating oxidative damage to both oligodendrocytes and neurons [36]. Sources of ROS/RNS are primarily cells of the innate immune system, in particular infiltrating macrophages and activated microglia cells, which release oxidative metabolites in the course of an "oxidative burst". In this context, it should be noted that chronic lesions also show pronounced signs of oxidative damage, although there are significantly fewer infiltrating immune cells present in these lesions. This indicates the presence of oxidative amplification mechanisms in the affected nervous system.

One of these amplifiers is progressive mitochondrial dysfunction, which generates additional free radicals in neurons and oligodendrocytes [37]. These free radicals in turn increase mitochondrial damage, thus creating an "oxidative vicious circle" that is further amplified by iron ions accumulating in the aging brain, especially in oligodendrocytes, as well as in macrophages and microglia cells during inflammatory processes. While iron ions are bound intracellularly to ferritin, thus largely suppressing their toxic effect, the death of these cells leads to the release of unbound $\mathrm{Fe}^{2+}$ into the extracellular space [8]. Due to the Fenton reaction (during which $\mathrm{Fe}^{2+}$ is transformed into $\mathrm{Fe}^{3+}$ ), iron ions have the ability to actively promote the formation of highly active hydroxyl radicals [38]. This reaction plays a central role in the pathophysiology of diseases such as hemochromatosis, resulting from severe overloading of organs with iron. Such iron deposits are also found in the margins of chronic-active MS lesions, especially in macrophages and microglia cells. If these lesions are followed over time using high-field MRI examinations, it can be seen that lesions with such an outer "iron ring" expand over time [39]. The fact that this increase in lesion volume is not observed in comparable lesions without an iron ring indicates that the iron deposit and release contribute to sustaining the chronic inflammatory reaction in the brain of MS patients.

\section{Glutamate and excitotoxicity}

Just as with the oxygen and nitrogen radicals previously discussed, the excitatory neurotransmitter glutamate can also be released by cells of the nervous system as well as by immune cells. Histopathological studies and CSF analyses as well as molecular biological and in vivo magnetic resonance spectroscopy (MRS) studies show that MS patients suffer from disturbances in brain glutamate homeostasis. Particularly active lesions, but also normal-appearing white matter show increased glutamate concentrations, which were found in a prospective MRS study to be predictive of the extent of neuroaxonal damage [40]. However, neither MR studies nor the increased concentrations found in CSF analysis can provide evidence of the exact origin and distribution of glutamate. At least in the context of acute inflammatory lesions, immune cells are regarded as an important source of glutamate and can release it via the cystine glutamate antiporter $\left(\mathrm{X}_{\mathrm{c}}{ }^{-}\right)[41]$; in an animal model, inhibition of this antiporter has a positive effect on the course of neuroinflammation [42]. In addition to the release of glutamate from immune cells, there is also experimental evidence for increased presynaptic release of this neurotransmitter from nerve cells under inflammatory attack [41]. At the same time, the uptake of excess glutamate in glial cells mediated by the glutamate transport proteins EAAT1 and 2 appears to be reduced in the vicinity of MS lesions [43].

Mechanistically, there are different ways, in which increased amounts of extracellular glutamate can exert their toxic effect. Excitotoxicity, meaning neuronal over-excitation by increased concentrations of the transmitter, is a pathophysiological concept that has been shown to play a role in experimental models of neurodegeneration and stroke. Unfortunately, however, these promising experimental findings have so far not been translated into successful clinical trials. In animal models of MS, a protective effect could also be achieved by blocking various synaptic glutamate receptors [44]. In principle, it is assumed that increased extracellular glutamate levels lead to an increased postsynaptic transmission, and cell damage is caused by the accompanying $\mathrm{Ca}^{2+}$ influx as well as the increased energetic burden of the neuron (see $>$ Fig. 3 ). Since glutamate receptors are preferably located postsynaptically, this mechanism could particularly contribute to synaptic pathology in the gray matter. Glutamate receptors have in addition been described on the membrane of axons, which supports the idea that they may also be a contributing factor to damage of the fiber tracts of the white matter [45]. Furthermore glutamate influences not only nerve cells but also affects the communication of immune cells, regulation of the blood-brain barrier as well as the interaction of axon and myelin [46,47]. Within the context of multiple sclerosis, this continues to make glutamate an interesting target structure for therapy, but a better understanding of the complex metabolism and pleiotropic mechanisms of glutamate in an inflammatory CNS milieu is necessary prior to the development of rational therapeutic strategies [48].

\section{Neuroglial Unit out of Equilibrium}

Neurons and oligodendrocytes form the structurally and functionally coupled axon-myelin unit. In the case of multiple sclerosis, damage to this unit is primarily triggered by the inflammatory reaction. However, there are also intrinsic factors within this highly regulated and efficient system that can increase or at least promote damage (see $>$ Fig. 3). These inherent weaknesses are often also affected by neurodegenerative pathologies and can develop their own detrimental dynamics over time.

\section{Oligodendrocyte damage und neuronal consequences}

The toxic environment of acute and chronic inflammatory activity often induces structural and molecular adaptation processes on the 
part of CNS cells, which primarily serve to counteract acute functional impairment, but in the long run can further promote the degenerative process. Such maladaptation also takes a central position in the concept of chronic axon damage by "virtual hypoxia". This concept describes how an imbalance of energy production and consumption can occur in inflammatory lesions, even without a lack of oxygen as e. g., in ischemia [49]. The starting point is the special anatomy of the white matter, in which myelination of the axons creates saltatory conduction resulting in acceleration of signal transmission. At the same time, energy consumption is optimized by a high density of ion channels at the nodes of Ranvier. Chronically demyelinated axons attempt to maintain propagation of action potentials along the axon by means of a new distribution of the ion channels, in particular voltage-dependent sodium channels, thus resulting in an increased influx of sodium. However, now more energy is required to ensure sufficient sodium export across the membrane. Due to increasing damage to ATP-producing mitochondria (see below) this increased energy requirement is accompanied by decreased energy production. A relative shortage of energy arises in the axon, in turn leading to the degeneration of axons over time.

Not only demyelination, being the complete loss of the myelin layers, has fatal consequences for the affected axons. Even a far more subtle damage to the axon-myelin unit can be dangerous for the axon. This is because the function of the myelin sheaths goes far beyond a passive, insulating role. Axon, myelin and oligodendrocytes should be understood as a closely linked, metabolic unit [50] containing small cytoplasmic channels that form in the myelin, which provide an important access path for macromolecules to the periaxonal space (the area between the axonal membrane and the innermost myelin layer). This is where the metabolic coupling of glia and neuron takes place. Lactate, produced by anaerobic glycolysis in oligodendrocytes, is supplied to the axon for further catabolism [51]. Glutamate released from the axon is a measurement parameter for the level of neuronal activity that in turn feeds back to the glucose metabolism machinery in the oligodendrocyte [47]. This metabolic coupling may, for example, already be disturbed when the structural integrity of the axon myelin unit is compromised even without a complete loss of the myelin sheath. The metabolic dependence of axons on this pathway is demonstrated by experimental studies in genetically modified mice that have collapsed myelin channels due to an altered molecular composition of their myelin. These mice develop primary axonal degeneration in the course of time; however this can be at least partially prevented by a further genetic modification of the myelin, which reopens these channels [52].

\section{Compromised mitochondrial distribution and function}

Mitochondria play a key role in the acute and chronic damage to neurons [53]. Morphological as well as biochemical and molecular biological analyses demonstrate that mitochondria in MS-affected tissue are impaired. In particular, oxidative phosphorylation, the central process of energy production, is affected by these changes - another potentially promotive factor for "virtual hypoxia" and chronic axonal damage [54]. In acute, inflammatory lesions, the process of mitochondrial damage is triggered by soluble mediators released by immune cells [35]. In the chronic disease stage, an accumulation of deletions in the mitochondrial genome could also be de- tected, which cause mitochondrial dysfunction and could contribute to progressive neurodegeneration [55]. This damage to mitochondria occurs not only in neurons but also affects oligodendrocytes and their precursor cells, which impairs the ability of the precursor cells to differentiate to myelinating oligodendrocytes and could thereby contribute to remyelination failure [56]. In addition to the structural and functional damage of mitochondria, their transport along the axons is diminished in response to inflammation, resulting in an accumulation of mitochondria, especially in regions of active infiltration. Such an impaired transport from the cell soma also results in a shortage of organelles in the neuronal periphery, which likely leads to a metabolic deficit at the synapse level. Remarkably, in experimental models of MS these transport deficiencies affect almost all axons in and around an inflammatory lesion and remain, at least in chronic models, for an extended period [57].

\section{Controversies and Perspectives}

Despite our improved knowledge of the pathology of progressive MS as well as our extended understanding of the pathogenetic processes that contribute to progression, central questions about the development, course and therapy remain unclear. This is also due to the fact that we know little with sufficient certainty: there is no clear knowledge about the genetic basis of progression, there are no truly validated animal models of progressive MS and no efficient therapies which provide us with clear pathogenetic hints. Nevertheless, in the following sections we will try to define some key issues about progressive MS and discuss the conclusions that can be drawn from the currently available data.

\section{Inflammation or Neurodegeneration - What Drives Progression?}

This is certainly the crucial question in MS research and good arguments can be found for both sides. In cases of progressive MS (as described above) there are indications of chronic inflammatory processes (such as in chronic active lesions or in the meninges) as well as evidence of prominent degenerative processes, which lead to a loss of axons and synapses. Furthermore, in experimental models secondary neurodegeneration can be evoked by primary inflammatory processes as well as secondary neuroinflammation triggered by primary neurodegenerative processes [58]. One possibility to approach the issue is to break it down into sub-questions. Thus, one can first ask, which imaging parameter correlates best with clinical progression. The answer to this is: atrophy of gray matter in the brain and spinal cord. Various MRI-based studies show that atrophy of the gray matter is particularly pronounced in progressive MS patients, the degree of which correlates with the extent of physical and cognitive impairment. Likewise it aids in predicting the likelihood of a clinical conversion from relapsing-remitting to progressive MS [59]. The next sub-question thus concerns the pathological changes underlying the decrease in gray matter volume. This question was answered in a study by Popescu and colleagues by parallel analysis of MS tissue using MRI and histology. The results showed that parameters of neuro-axonal damage (such as axon density as well as the size and number of neuronal cell bodies), but not the extent of the demyelination, determine the corti- 
cal atrophy measured in the MRI [22]. Together with recent findings, which indicate a widespread loss of synapses in the gray matter of progressive MS patients [23,24], this suggests that the diffuse degeneration of neuronal elements (nerve cells, axons and synapses) causes the loss of gray matter volume. The next questions is: what is the cause of this diffuse neuronal degeneration? In principle, three answers should be considered: (1) A primary neurodegenerative process, (2) secondary neurodegeneration as a result of chronic inflammatory lesions of the white matter or (3) local inflammatory damage to neurons. A primary neurodegenerative process appears to be an unlikely cause, not least for the following reasons. First, in its genetics MS more closely resembles an autoimmune disease than a neurodegenerative disorder [60]. Second, pathological studies demonstrate the presence of an inflammatory response in progressive MS, which differs quantitatively and qualitatively from inflammatory processes in classical neurodegenerative diseases [61]. Finally, highly effective immunosuppressive therapies reduce not only the risk of relapse but also the likelihood of increased disability [62]. If primary neurodegeneration is ruled out as a cause, then two inflammation-related processes remain. One explanation is that inflammatory lesions in the white matter lead to the degeneration of neuronal connections, the disappearance of which in turn causes progressive atrophy or degeneration in the gray matter. In principle, this degeneration could be both retrograde - in which case the projection neurons axotomised in the lesion would be affected - as well as anterograde and possibly transsynaptic - in this case the target cells of the interrupted connections would be primarily affected. A finding which is compatible with the presence of such a propagated degeneration relates to the fact that cortical atrophy occurs in a non-random, regionally accentuated pattern in multiple sclerosis [63], that is, highly connected cortical centers ("hubs") are particularly affected by the degenerative process. However, such regional concentration could also have other causes such as regional inflammatory pathology (e.g., the local presence of meningeal follicles). Furthermore, in a predominantly propagated degenerative mechanism, a closer correlation between the number of lesions of the white matter and the extent of gray matter atrophy would be expected [64] and one would assume that anti-inflammatory therapies that prevent the occurrence of new lesions in the white matter would show more pronounced effects on the clinical course of the disease and atrophy up to the progressive stage of the disease [65]. In short, neuronal degeneration in the gray matter based solely on propagated degeneration seems less probable. On the other hand, from our point of view, there is evidence that local inflammatory processes are decisive factors in this neurodegenerative process. Pathological studies show that even in the progressive stage of multiple sclerosis, inflammatory processes can occur in the meninges and gray matter, the presence of which correlates with the extent of neuronal degeneration as well as the clinical progression of the disease $[16,17,19]$. These findings have also been supported by recent PET studies. The ligands used show increased microglial activation, especially in the gray matter of progressive MS patients, the extent of which is correlated with the degree of neurological deterioration and cognitive function impairment [10].
Primary and Secondary Progressive MS - 2 Different Disease Entities or 2 Sides of the Same Coin?

Another interesting question relates to the utility of the traditional classification of progressive MS, which subdivides into a secondary progressive (SPMS) and a primary progressive (PPMS) course. The former is preceded by an early relapsing-remitting phase, while this phase is absent in the latter. This is not just a semantic issue, but a clinically important discussion. The answer, for example, determines whether clinical trials must be carried out separately or are mutually transferable in both progressive forms.

First, a number of findings suggest that both forms of progressive MS are at least part of the same disease spectrum. Thus, relatives of patients who are suffering from a classical relapsing-remitting form of MS have not only an increased risk for this subtype of disease but also for the occurrence of primary progressive MS [66]. Furthermore, a recent long-term observation of patients with a radiologically isolated syndrome (RIS) shows that the patient can develop both an relapsing-remitting form of MS as well as a primary progressive form of the disease [67]. Likewise, analyses of the pathology indicate that the differences between primary and secondary progressive MS are more quantitative than qualitative $[61,68]$. It is important to note that PPMS patients also exhibit focal white matter lesions, which we will take as a starting point for a short thought experiment: Let us assume that clinically apparent relapses are caused by focal lesions of the white matter. However, not all of these lesions also produce a relapse, since only lesions that are located in clinically particularly eloquent areas (for example in the optic nerve, cerebellum or brainstem) also lead to clinically detectable symptoms. Thus both PPMS patients and SPMS patients have focal lesions in the white matter, but these 2 groups of patients differ primarily in the random distribution of their lesions to clinically more or less eloquent areas. In short and more provocatively put: PPMS is nothing more than SPMS with a clinically silent relapsing-remitting phase. With respect to their pathogenetic bases, the assignment to the categories SPMS and PPMS appears to be rather arbitrary and of little significance. In fact, in the most recent classification of progressive MS, more objective criteria are increasingly in the forefront $[1,69]$. For example both the presence of relapses as well as new lesions detected in MRI result in classification as active MS with progression $[1,69]$.

\section{Treatment for Progressive MS: Nihilism or Optimism?}

The crucial question for affected MS patients is now whether new pathological findings and pathogenetic concepts can also be translated into successful treatment for patients with progressive MS. This is particularly important because numerous studies in recent years show that many immunomodulatory or immunosuppressive therapeutic approaches, which are very successful in the relapsing-remitting phase of MS, do not show comparable efficiency in the progressive phase of the disease (see $>$ Table 1). Based on the expanded understanding of the pathogenesis of progressive MS, therapeutic approaches relying on the following strategies appear to be particularly promising: A first approach takes into account immunomodu- 
- Table 1 Selection of ongoing and closed phase III studies of progressive multiple sclerosis.

\begin{tabular}{|c|c|c|c|c|c|c|c|}
\hline Studies & Drug & Status & $\begin{array}{l}\text { Popula- } \\
\text { tion }\end{array}$ & Follow-up & Primary endpoint & $\begin{array}{l}\text { Endpoint } \\
\text { achieved }\end{array}$ & Results \\
\hline ASCEND & Natalizumab & Discontinued & SPMS & 96 weeks & 6-month CDW & No & $\begin{array}{l}\text { OR } 0.86 \\
(0.66-1.13)\end{array}$ \\
\hline CUPID & Dronabinol & Completed & $\begin{array}{l}\text { PPMS and } \\
\text { SPMS }\end{array}$ & 3 years & 6-month CDW & No & $\begin{array}{l}\text { HR } 0.92 \\
(0.68-1.23)\end{array}$ \\
\hline EXPAND & Siponimod & $\begin{array}{l}\text { Completed test } \\
\text { phase }\end{array}$ & SPMS & 3 years & 3-month CDW & Yes & HR 0.79 \\
\hline INFORMS & Fingolimod & Completed & PPMS & 3 years & 3-month CDW & No & $\begin{array}{l}\text { HR } 0.95 \\
(0.80-1.12)\end{array}$ \\
\hline MS-SPI & Biotin (MD1003) & Completed & $\begin{array}{l}\text { PPMS and } \\
\text { SPMS }\end{array}$ & 1 year & $\begin{array}{l}\text { 12-month } \\
\text { improvement } \\
\text { (confirmed) }\end{array}$ & Yes & $\begin{array}{l}12.6 \% \\
\text { improvement } \\
\text { biotin vs. } 0 \% \\
\text { placebo (SPI 1) }\end{array}$ \\
\hline MS-SPI2 & Biotin (MD1003) & Recruited & $\begin{array}{l}\text { PPMS and } \\
\text { SPMS }\end{array}$ & 15 months & $\begin{array}{l}\text { 15-month } \\
\text { improvement } \\
\text { (confirmed) }\end{array}$ & NA & NA \\
\hline OLYMPUS & Rituximab & Completed & PPMS & 96 weeks & 3-month CDW & No & $\begin{array}{l}\text { HR } 0.77 \\
(0.55-1.09)\end{array}$ \\
\hline ORATORIO & Ocrelizumab & Completed & PPMS & 120 weeks & 3-month CDW & Yes & $\begin{array}{l}\text { HR } 0.76 \\
(0.59-0.98)\end{array}$ \\
\hline
\end{tabular}

$\mathrm{CDW}=$ Confirmed Disability Worsening, $\mathrm{OR}=$ Odds Ratio, $\mathrm{HR}=$ Hazard Ratio, SPMS = Secondary Progressive Multiple Sclerosis, PPMS = Primary Progressive Multiple Sclerosis, NA = No data available For source and additional information, see [1] and clinicaltrials.gov.

lating therapeutic strategies specifically tailored to the inflammatory response in progressive MS. The starting point of this "immunomodulation 2.0" could include B-cell-rich meningeal infiltrates and follicles as well as the activation of mononuclear phagocytes (i. e., macrophages and microglia cells) in the CNS parenchyma. In principle, anti-inflammatory therapies can also be effective in the progressive phase of the disease as has been demonstrated by the positive results of phase III studies of ocrelizumab, a B-cell-depleting antibody in primary progressive MS patients as well as siponimod, a selective S1P1 and S1P5 agonist tested in MS patients with secondary progressive disease (see $>$ Table 1 ). However, it should be noted that the observed therapeutic effects were comparatively mild in both studies, and that especially younger patients with relatively high inflammatory activity might particularly benefit. Intrathecal administration might be a comparatively direct means to focus the effects of anti-inflammatory strategies on the CNS. A preliminary study of intrathecal administration of a B-cell-depleting antibody in patients with progressive multiple sclerosis has shown that in fact, increased antibody concentrations in the CSF could be achieved over several months, but this led only to an insufficient and time-limited depletion of B cells in this compartment [70].

A second approach, which is based on the current concepts of pathogenesis, is the attempt to support the nervous system itself, which has been thrown out of balance by the chronic inflammatory reaction. So far, attempts have been made to improve the ionic disequilibrium, e. g., by administering sodium channel blockers or cannabinoids. However, these approaches have largely been disappointing in clinical implementation [1]. It is however conceivable that the main target of these therapeutic approaches - dam- age to chronically demyelinated axons in the white matter - plays a less important role in the progressive phase of the disease compared to damage to the gray matter. Support for this interpretation comes from a study of patients with acute optic neuritis, which demonstrated that sodium channel blockers can at least partially prevent axonal damage in active white matter lesions in humans and therefore should preferably be used in an earlier stage of the disease [71]. An attempt to offset the energetic imbalance by the administration of a high dose of the vitamin biotin was clinically so far more promising. This initial study of 103 patients with primary or secondary progressive MS showed a clinical improvement in $12.6 \%$ of the subjects treated (no corresponding recovery was observed in the 51-patient control group [72]). Even though these results may be reason for cautious optimism, it is important that they are reproduced in a larger ongoing study. The further development of this therapeutic concept is further dependent on the more exact characterization of the possible effect of biotin, which is primarily an important coenzyme of carboxylase enzymes.

Considering the close metabolic coupling between the axon and the myelin layer, therapeutic approaches for the promotion of remyelination can also be understood as an attempt to restore energetic balance. However, these therapeutic strategies are primarily directed toward focal lesions of the white matter, whether they involve administration of a neutralizing antibody against the remyelination inhibitor LINGO-1 [73] or the use of a remyelination-promoting drug such as clemastine [74]. Accordingly, it remains open to what extent these treatment strategies ultimately affect the diffuse neuronal degeneration processes in the gray matter. Comparatively less noted and less studied strategies that might possibly 
affect these neural degeneration processes, however, are rehabilitation measures, such as an increase in physical activity [75]. Experimental studies indicate that an increase in physical activity improves the function and maintenance of the synapses. For example, clinical pilot studies suggest positive effects on the cognitive performance of patients with progressive MS [75]. However, further experimental and clinical trials are urgently needed to better assess the extent to which MS patients can "run away" from the progression of their disease.

\section{Summary}

Multiple sclerosis changes not only its clinical appearance, but also its pathological and pathomechanistic characteristics when transitioning from the relapsing-remitting phase into the progressive phase. While focal lesions of the white matter are predominant in the RR phase, diffuse changes of gray and white matter determine the pathology of progressive MS. In this stage, the drivers of the disease are no longer migrating immune cells from the periphery, but a chronic inflammatory reaction, which spreads, largely decoupled from the peripheral immune system, in the damaged central nervous system. Therapeutic strategies are therefore particularly promising if they can curb this inflammatory reaction locally in the brain and spinal cord or restore the resilience of the structurally and functionally impaired nervous system.

\section{Acknowledgement}

Translated from the original German by Kevin Fulton.

\section{Conflict of Interest}

In the past, M. Kerschensteiner has received honoraria for consulting and presentations from Biogen, Genzyme/Sanofi-Aventis, MedDay Pharmaceuticals, Novartis and TEVA, as well as research support from Biogen and Genzyme/Sanofi-Aventis. The other authors declare they have no conflicts of interest.

\section{Literatur}

[1] Ontaneda D, Thompson AJ, Fox RJ et al. Progressive multiple sclerosis: prospects for disease therapy, repair, and restoration of function. Lancet 2017; 389: 1357-1366

[2] Charcot J-M. Histologie de la sclérose en plaques. 1868;

[3] Compston A, Coles A. Multiple sclerosis. Lancet 2008; 372: 1502-1517

[4] Frischer JM, Weigand SD, Guo Y et al. Clinical and pathological insights into the dynamic nature of the white matter multiple sclerosis plaque. Ann Neurol 2015; 78: 710-721

[5] Absinta M, Sati P, Schindler M et al. Persistent 7-tesla phase rim predicts poor outcome in new multiple sclerosis patient lesions. J Clin Invest 2016; 126: 2597-2609

[6] Goldschmidt T, Antel J, König FB et al. Remyelination capacity of the MS brain decreases with disease chronicity. Neurology 2009; 72: 1914-1921

[7] Miron VE, Boyd A, Zhao J-W et al. M2 microglia and macrophages drive oligodendrocyte differentiation during CNS remyelination. Nat Neurosci 2013; 16: 1211-1218
[8] Lassmann H, van Horssen J, Mahad D. Progressive multiple sclerosis: pathology and pathogenesis. Nat Rev Neurol 2012; 8: 647-656

[9] Kutzelnigg A, Lucchinetti CF, Stadelmann C et al. Cortical demyelination and diffuse white matter injury in multiple sclerosis. Brain 2005; 128: $2705-2712$

[10] Herranz E, Giannì C, Louapre C et al. Neuroinflammatory component of gray matter pathology in multiple sclerosis. Ann Neurol 2016; 80: 776-790

[11] Kutzelnigg A, Lassmann $\mathrm{H}$. Cortical demyelination in multiple sclerosis: A substrate for cognitive deficits? J Neurol Sci 2006; 245: 123-126

[12] Haider L, Zrzavy T, Hametner S et al. The topograpy of demyelination and neurodegeneration in the multiple sclerosis brain. Brain 2016; 139: $807-815$

[13] Vercellino M, Plano F, Votta B et al. Grey matter pathology in multiple sclerosis. J Neuropathol Exp Neurol 2005; 64: 1101-1107

[14] Gilmore CP, Bö L, Owens T et al. Spinal cord gray matter demyelination in multiple sclerosis-a novel pattern of residual plaque morphology. Brain Pathol 2006; 16: 202-208

[15] Peterson JW, Bö L, Mörk S et al. Transected neurites, apoptotic neurons, and reduced inflammation in cortical multiple sclerosis lesions. Ann Neurol 2001; 50: 389-400

[16] Howell OW, Reeves CA, Nicholas R et al. Meningeal inflammation is widespread and linked to cortical pathology in multiple sclerosis. Brain 2011; 134: 2755-2771

[17] Magliozzi R, Howell OW, Reeves C et al. A Gradient of neuronal loss and meningeal inflammation in multiple sclerosis. Ann Neurol 2010; 68: 477-493

[18] Fischer MT, Wimmer I, Höftberger R et al. Disease-specific molecular events in cortical multiple sclerosis lesions. Brain 2013; 136: 1799-1815

[19] Magliozzi R, Howell O, Vora A et al. Meningeal B-cell follicles in secondary progressive multiple sclerosis associate with early onset of disease and severe cortical pathology. Brain 2007; 130: 1089-1104

[20] Damjanovic D, Valsasina P, Rocca MA et al. Hippocampal and deep gray matter nuclei atrophy is relevant for explaining cognitive impairment in MS: A multicenter study. AJNR Am J Neuroradiol 2017; 38: $18-24$

[21] Schlaeger R, Papinutto N, Zhu AH et al. Association between thoracic spinal cord gray matter atrophy and disability in multiple sclerosis. JAMA Neurol 2015; 72: 897-904

[22] Popescu V, Klaver R, Voorn P et al. What drives MRI-measured cortical atrophy in multiple sclerosis? Mult Scler 2015; 21: 1280-1290

[23] Jürgens T, Jafari M, Kreutzfeldt M et al. Reconstruction of single cortical projection neurons reveals primary spine loss in multiple sclerosis. Brain 2016; 139: 39-46

[24] Albert M, Barrantes-Freer A, Lohrberg M et al. Synaptic pathology in the cerebellar dentate nucleus in chronic multiple sclerosis. Brain Pathol 2016, doi:10.1111/bpa.12450

[25] Kreutzfeldt M, Bergthaler A, Fernandez M et al. Neuroprotective intervention by interferon- $\mathrm{y}$ blockade prevents CD8 + T cell-mediated dendrite and synapse loss. J Exp Med 2013; 210: 2087-2103

[26] Rossi S, Motta C, Studer V et al. Tumor necrosis factor is elevated in progressive multiple sclerosis and causes excitotoxic neurodegeneration. Mult Scler 2014; 20: 304-312

[27] Gardner C, Magliozzi R, Durrenberger PF et al. Cortical grey matter demyelination can be induced by elevated pro-inflammatory cytokines in the subarachnoid space of MOG-immunized rats. Brain 2013; 136 : 3596-3608

[28] Merkler D, Ernsting T, Kerschensteiner M et al. A new focal EAE model of cortical demyelination: Multiple sclerosis-like lesions with rapid resolution of inflammation and extensive remyelination. Brain 2006; 129: $1972-1983$

[29] Zamanian JL, Xu L, Foo LC et al. Genomic analysis of reactive astrogliosis. J Neurosci 2012; 32: 6391-6410 
[30] Liddelow SA, Guttenplan KA, Clarke LE et al. Neurotoxic reactive astrocytes are induced by activated microglia. Nature 2017; 541 : 481-487

[31] Mayo L, Trauger SA, Blain M et al. Regulation of astrocyte activation by glycolipids drives chronic CNS inflammation. Nat Med 2014; 20: 1147-1156

[32] Habbas S, Santello M, Becker D et al. Neuroinflammatory TNFa impairs memory via astrocyte signaling. Cell 2015; 163: 1730-1741

[33] Schafer DP, Lehrman EK, Kautzman AG et al. Microglia sculpt postnatal neural circuits in an activity and complement-dependent manner. Neuron 2012; 74: 691-705

[34] Watkins LM, Neal JW, Loveless S et al. Complement is activated in progressive multiple sclerosis cortical grey matter lesions. J Neuroinflammation 2016; 13: 161

[35] Nikić I, Merkler D, Sorbara C et al. A reversible form of axon damage in experimental autoimmune encephalomyelitis and multiple sclerosis. Nat Med 2011; 17: 495-499

[36] Haider L, Fischer MT, Frischer JM et al. Oxidative damage in multiple sclerosis lesions. Brain 2011; 134: 1914-1924

[37] Murphy MP. How mitochondria produce reactive oxygen species. Biochem J 2009; 417: 1-13

[38] Prousek J. Fenton chemistry in biology and medicine. J Macromol Sci Part A Pure Appl Chem 2007; 79: 2325

[39] Dal-Bianco A, Grabner G, Kronnerwetter C et al. Slow expansion of multiple sclerosis iron rim lesions: Pathology and 7T magnetic resonance imaging. Acta Neuropathol 2017; 133: 25-42

[40] Azevedo C], John K, Philip C et al. In vivo evidence of glutamate toxicity in multiple sclerosis. Ann Neurol 2014; 76: 269-278

[41] Macrez R, Stys PK, Vivien D et al. Mechanisms of glutamate toxicity in multiple sclerosis: Biomarker and therapeutic opportunities. Lancet Neurol 2016; 15: 1089-1102

[42] Evonuk KS, Baker B], Doyle RE et al. Inhibition of system Xc(-) transporter attenuates autoimmune inflammatory demyelination. J Immunol 2015; 195: 450-463

[43] Pitt D, Nagelmeier IE, Wilson HC et al. Glutamate uptake by oligodendrocytes: Implications for excitotoxicity in multiple sclerosis. Neurology 2003; 61: 1113-1120

[44] Sulkowski G, Dabrowska-Bouta B, Chalimoniuk M et al. Effects of antagonists of glutamate receptors on pro-inflammatory cytokines in the brain cortex of rats subjected to experimental autoimmune encephalomyelitis. J Neuroimmunol 2013; 261: 67-76

[45] Ouardouz M, Coderre E, Basak A et al. Glutamate receptors on myelinated spinal cord axons: I. GluR6 kainate receptors. Ann Neurol 2009; 65: 151-159

[46] Affaticati P, Mignen O, Jambou F et al. Sustained calcium signalling and caspase-3 activation involve NMDA receptors in thymocytes in contact with dendritic cells. Cell Death Differ 2011; 18: 99-108

[47] Saab AS, Tzvetavona ID, Trevisiol A et al. Oligodendroglial NMDA receptors regulate glucose import and axonal energy metabolism. Neuron 2016; 91: 119-132

[48] Hohlfeld R, Kerschensteiner M. Antiglutamatergic therapy for multiple sclerosis? Lancet Neurol 2016; 15: 1003-1004

[49] Trapp BD, Stys PK. Virtual hypoxia and chronic necrosis of demyelinated axons in multiple sclerosis. Lancet Neurol 2009; 8: 280-291

[50] Simons M, Misgeld T, Kerschensteiner M. A unified cell biological perspective on axon-myelin injury. J Cell Biol 2014; 206: 335-345

[51] Fünfschilling U, Supplie LM, Mahad D et al. Glycolytic oligodendrocytes maintain myelin and long-term axonal integrity. Nature 2012; 485: 517-521

[52] Snaidero N, Velte C, Myllykoski M et al. Antagonistic functions of MBP and CNP establish cytosolic channels in CNS myelin. Cell Rep 2017; 18: 314-323

[53] Witte ME, Mahad D], Lassmann $\mathrm{H}$ et al. Mitochondrial dysfunction contributes to neurodegeneration in multiple sclerosis. Trends Mol Med 2014; 20: 179-187
[54] Mahad D], Ziabreva I, Campbell G et al. Mitochondrial changes within axons in multiple sclerosis. Brain 2009; 132: 1161-1174

[55] Campbell GR, Ziabreva I, Reeve AK et al. Mitochondrial DNA deletions and neurodegeneration in multiple sclerosis. Ann Neurol 2011; 69: 481-492

[56] Ziabreva I, Campbell G, Rist J et al. Injury and differentiation following inhibition of mitochondrial respiratory chain complex IV in rat oligodendrocytes. Glia 2010; 58: 1827-1837

[57] Sorbara CD, Wagner NE, Ladwig A et al. Pervasive axonal transport deficits in multiple sclerosis models. Neuron 2014; 84: 1183-1190

[58] Hemmer B, Kerschensteiner M, Korn T. Role of the innate and adaptive immune responses in the course of multiple sclerosis. Lancet Neurol 2015; 14: 406-419

[59] Calabrese M, Romualdi C, Poretto $V$ et al. The changing clinical course of multiple sclerosis: a matter of gray matter. Ann Neurol 2013; 74: 76-83

[60] Farh KK-H, Marson A, Zhu J et al. Genetic and epigenetic fine mapping of causal autoimmune disease variants. Nature 2015; 518: 337-343

[61] Frischer JM, Bramow S, Dal-Bianco A et al. The relation between inflammation and neurodegeneration in multiple sclerosis brains. Brain 2009; 132: 1175-1189

[62] Jokubaitis VG, Spelman T, Kalincik T et al. Predictors of long-term disability accrual in relapse-onset multiple sclerosis. Ann Neurol 2016; 80: $89-100$

[63] Steenwijk MD, Geurts JJG, Daams M et al. Cortical atrophy patterns in multiple sclerosis are non-random and clinically relevant. Brain 2016; 139: $115-126$

[64] Bermel RA, Bakshi R. The measurement and clinical relevance of brain atrophy in multiple sclerosis. Lancet Neurol 2006; 5: 158-170

[65] Ontaneda D, Fox RJ, Chataway J. Clinical trials in progressive multiple sclerosis: lessons learned and future perspectives. Lancet Neurol 2015; 14: 208-223

[66] Weinshenker BG, Bulman D, Carriere W et al. A comparison of sporadic and familial multiple sclerosis. Neurology 1990; 40: 1354-1358

[67] Kantarci OH, Lebrun C, Siva A et al. Primary progressive multiple sclerosis evolving from radiologically isolated syndrome. Ann Neurol 2016; 79: 288-294

[68] Frischer JM, Weigand SD, Guo Y et al. Clinical and pathological insights into the dynamic nature of the white matter multiple sclerosis plaque. Ann Neurol 2015; 78: 710-721

[69] Lublin FD, Reingold SC, Cohen JA et al. Defining the clinical course of multiple sclerosis: the 2013 revisions. Neurology 2014; 83: 278-286

[70] Komori M, Lin YC, Cortese I et al. Insufficient disease inhibition by intrathecal rituximab in progressive multiple sclerosis. Ann Clin Transl Neurol 2016; 3: 166-179

[71] Raftopoulos R, Hickman S], Toosy A et al. Phenytoin for neuroprotection in patients with acute optic neuritis: A randomised, placebo-controlled, phase 2 trial. Lancet Neurol 2016; 15: 259-269

[72] Tourbah A, Lebrun-Frenay C, Edan G et al. MD1003 (high-dose biotin) for the treatment of progressive multiple sclerosis: A randomised, double-blind, placebo-controlled study. Mult Scler 2016; 22: 1719-1731

Cadavid DB. Efficacy analysis of the Anti-LINGO-1 monoclonal antibody BIIB033 in acute optic neuritis: The RENEW trial. Neurology 84 (Suppl 14): P7202 2015

[73] Green A, Gelfand J, Cree G et al. Positive phase II double-blind randomized placebo-controlled crossover trial of clemastine fumarate for remyelination of chronic optic neuropathy in MS. Annual Meeting of the American Academy of Neurology; Vancouver, BC, Canada abstr ES1008 2016.

[74] Campbell E, Coulter EH, Mattison PG et al. Physiotherapy rehabilitation for people with progressive multiple sclerosis: A systematic review. Arch Phys Med Rehabil 2016; 97: 141-151.e143 